\title{
ÉTICA Y DERECHOS EN LA UNIVERSIDAD DEL
}

\section{SIGLO XXI}

Prof. Dr. Emilio Martínez Navarro

Universidad de Murcia

\section{INTRODUCCIÓN}

Los términos «ética»y «derechos»se prestan a diversos malentendidos, porque son vocablos de uso muy frecuente en el lenguaje cotidiano y que tienen diversos significados que los hablantes pueden confundir entre sí. Por ello conviene, de entrada, aclarar en qué sentido vamos a utilizar aquí ambas expresiones.

El término «ética» lo vamos a utilizar, en el contexto de este artículo, en el sentido de un saber que la humanidad ha ido construyendo desde tiempos inmemoriales para orientar los comportamientos individuales y colectivos conforme a criterios de bondad y maldad. Este saber tiene dos niveles distintos, pero estrechamente conectados entre sí: la moral cotidiana y la reflexión ética.

La moral cotidiana se aprende sobre todo en la infancia, y contiene normas, preceptos y valores que la persona va interiorizando sin darse cuenta, dando lugar a una moralidad personal que sirve a cada cual para saber cuándo está actuando bien y cuándo

\footnotetext{
${ }^{1}$ Este artículo es un desarrollo y reelaboración de la ponencia presentada en el XVIII Encuentro Estatal de Defensores Universitarios, celebrado en la Universidad Europea de Madrid (Campus Villaviciosa de Odón) el 4 de noviembre de 2015. Agradezco a los organizadores de dicho Encuentro, y a todo el colectivo de Defensores Universitarios, tanto la invitación como la cordial acogida que me dispensaron.
} 
actúa mal, y también sirve para juzgar moralmente el comportamiento ajeno desde los mismos criterios personalmente asumidos. Tales criterios forman parte de la tradición cultural en la que cada cual haya sido educado. Naturalmente, esta moral personal cotidiana, tomada del propio grupo cultural, la vamos revisando y modificando a lo largo de la vida, pero cuando hacemos tal revisión entra en funcionamiento el otro nivel del saber ético: la reflexión ética.

La reflexión ética es filosofía sobre la moral. Todas las personas, en mayor o menor medida, practicamos este filosofar sobre los propios valores, normas y preceptos morales que nos han inculcado desde la niñez y que forman parte de alguna de las tradiciones culturales que ha atesorado la humanidad a lo largo de los siglos. Sin embargo, a partir del siglo V a.C., con el nacimiento de la Filosofía entre los antiguos griegos, la reflexión ética se convierte en «Ética», es decir, en la rama de la Filosofía que revisa de un modo racional y sistemático el saber moral cotidiano, con el fin de llevar a cabo tres tareas principales:

1) Aclarar en qué consiste la moralidad, es decir, distinguirla de otros saberes emparentados (como la religión, el derecho y las normas de protocolo social), señalar posibles contradicciones y tabúes obsoletos en el saber moral heredado, etc. Esta tarea de aclaración la desarrollan los filósofos de moral. A lo largo de los siglos se han propuesto diversas teorías éticas entre las que destacamos aquí sólo algunas: la ética de la virtud (Aristóteles), el deontologismo de Kant, el utilitarismo de John Stuart Mill y las éticas del diálogo de J. Habermas y K.-O. Apel.

2) Fundamentar racionalmente la moralidad, es decir, averiguar si tiene sentido mantener en uso los términos morales (como «deber», «virtud», «mentira», 
«injusticia», etc.). En caso afirmativo, la Ética ha de exponer las razones que avalan la racionalidad de lo moral. Pero en caso negativo, la Ética nos tendría que mostrar los argumentos por los cuales resulta absurdo mantener el lenguaje moral y por qué lo más sensato sería abandonarlo por completo. La mayor parte de los filósofos morales de todos los tiempos consideran que la moral tiene una base racional y que se trata de un saber del que no podemos prescindir, pero las diversas teorías éticas discrepan entre sí en cuanto al modo de entender dicho saber y cómo ha de articularse con los demás saberes.

3) Aplicar, a los casos de la vida cotidiana de las personas y de las instituciones, los principios y argumentos que se hayan podido elaborar en las dos tareas anteriores (aclaración y fundamentación), con el fin de orientar nuestra vida personal y colectiva en un horizonte de humanización. Es decir, se trata de aplicar a la práctica cotidiana una moralidad crítica, no meramente la moralidad «heredada» sin más, sino una moralidad depurada por la reflexión filosófica que pueden aportar especialistas (filósofos) y también no especialistas (personas reflexivas que, desde su ámbito particular de actividad, proponen valores, normas y preceptos para orientar ese ámbito para hacerlo más justo, más incluyente, más humanizador).

¿Dónde situamos la ética universitaria? Conforme a las distinciones conceptuales que acabamos de exponer, la ética universitaria es una más de las éticas aplicadas. Del mismo modo que hay una ética médica, una ética de la ingeniería, una ética del deporte, etc., hay también una ética universitaria que, conforme a los fundamentos teóricos que 
aquí presuponemos ${ }^{2}$, es fruto de la reflexión que llevan a cabo, de manera dialógica, un conjunto de personas que forman parte del ámbito universitario con asesoría de expertos en Filosofía Moral. Los contenidos (valores, normas y preceptos morales) de una posible ética universitaria han de ser fruto de una deliberación racional y razonable en la que participen, en pie de igualdad, los afectados por la institución universitaria (comúnmente llamados «grupos de interés») y los expertos en cuestiones éticas.

En el esquema siguiente recogemos las distinciones que hemos comentado hasta

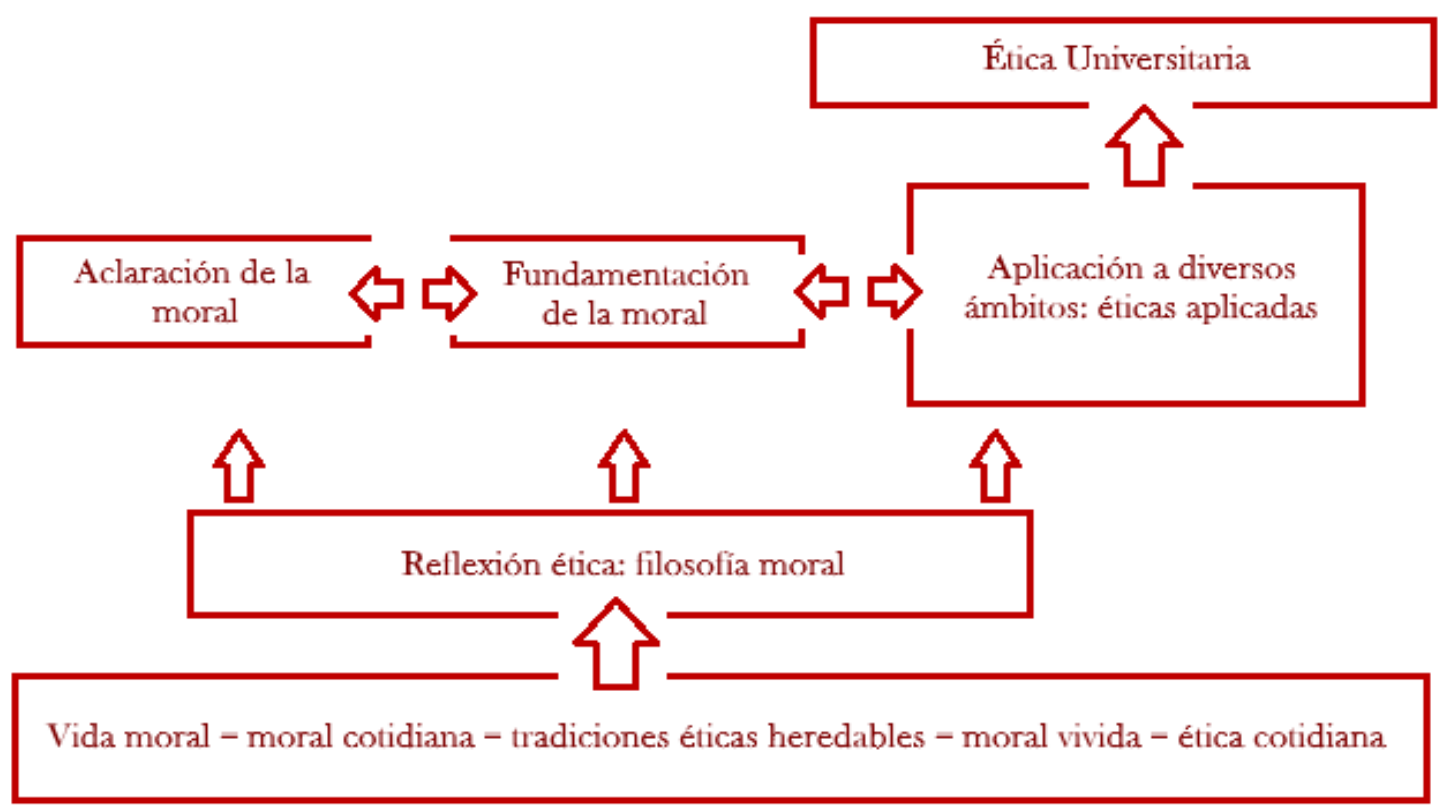

el momento (figura 1):

Figura 1: Esquema de los tipos de saber ético para situar la ética universitaria (elaboración propia).

\footnotetext{
${ }^{2}$ El marco teórico que subyace a las ideas aquí expuestas está expuesto con cierto detalle en obras como los siguientes: Cortina, A. y Martínez E. (1996) y Martínez, E. (2010).
} 
Con respecto al término «derechos», aquí lo vamos a utilizar con la significación de «protecciones legales sobre capacidades valiosas para una vida digna» (Cortina 2009, cap. 9). Esto significa que reconocemos unos derechos básicos a las personas en virtud de que se supone que tales derechos están definidos y establecidos por la comunidad para proteger algo especialmente valioso: las capacidades de las personas. En este sentido, los derechos proclamados en la Declaración Universal de los Derechos Humanos de la ONU (1948) y las demás declaraciones de la ONU, así como los derechos básicos recogidos en la legislación vigente, los entendemos aquí como exigencias ético-jurídicas válidas que cualquier persona puede alegar para ver protegido el ejercicio de sus capacidades básicas como ser humano. Los derechos son "cartas de triunfo" (R. Dworkin) en posesión de sus beneficiarios: son unos mínimos de justicia que han de ser respetados por todos en todo momento. Es conveniente que los reglamentos universitarios expresen con toda claridad qué derechos tiene cada cual y cuáles son los límites de cada derecho, para evitar colisiones entre diversos derechos. Muchos derechos llevan aparejado algún deber. Por ello es conveniente recordar también los deberes. Ahora bien, el lenguaje de los derechos y los deberes es más jurídico que ético, mientras que aquí nos vamos a centrar en las cuestiones de ética: Lo ético es el juego de la prevención de conflictos, sin tener que llegar al terreno del derecho, y también es el terreno de los argumentos racionales acerca de por qué consideramos que ciertos comportamientos son razonables, válidos y dignos de alabanza, mientras que otros son irrazonables, no válidos y dignos de rechazo.

\section{La ética de la institución universitaria entre las éticas aplicadas}

Hemos comentado anteriormente que la ética universitaria es una más de las éticas aplicadas. Cabe preguntarse ahora: ¿De qué modo (con qué método) se puede averiguar 
en cada una de las éticas aplicadas lo que es moralmente correcto y lo que no? Existen varios métodos (Cortina y Martínez 1996, cap. 6) para construir racionalmente una ética aplicada, pero el método que considero más razonable es el que propone Adela Cortina (1993) y que se llama «hermenéutica crítica de las actividades sociales». Este método propone que, en todas las actividades humanas en las que queramos averiguar cuáles son los principios éticos que le corresponden, conviene seguir dos momentos sucesivos:

1) El momento deontológico, que corresponde a los deberes básicos a tener en cuenta en todas las actividades humanas.

2) El momento de la buena praxis en cada actividad concreta.

En efecto, en un primer momento conviene reconocer que todas las actividades humanas han de respetar y promover unos mínimos básicos relacionados con la dignidad de las personas: los derechos humanos. Este primer componente de las éticas aplicadas es compartido por todas ellas. Se le puede llamar «el momento kantiano» porque sugiere que lo éticamente primero es el deber de tratar a las personas como fines en sí mismas, y no como meros medios instrumentalizables.

Por otro lado, el segundo momento de elaboración de las éticas aplicadas es el que aterriza en las cuestiones éticas específicas de cada ámbito concreto. A este segundo momento se le puede llamar «el momento aristotélico», porque en gran medida está inspirado en la noción de Aristóteles de lo que es «una práctica» (MacIntyre 1981), es decir, una actividad humana en la que participan cooperativamente distintas personas respetando ciertas reglas. A partir de esta idea, en la Ética contemporánea ha hecho fortuna una distinción conceptual que puede ser útil para comprender las bases morales de cualquier institución: se trata de la distinción entre los «bienes internos» y los «bienes 
externos». Los bienes internos son las metas, los fines, para los cuales nace la institución; son los objetivos que — se supone- que persigue la institución de que se trate. Por ejemplo, se supone que la profesión periodística es una institución social encargada de proporcionar al público información, opinión, entretenimiento, etc.; se supone que la profesión docente ha de proporcionar al alumnado una formación, adiestramiento, etc.; lo propio de la profesión médica sería la curación de enfermedades, la reparación de roturas, la prevención de enfermedades, los cuidados paliativos, etc.; lo específico de una organización empresarial dedicada, por ejemplo, a fabricar zapatos, sería ofrecer zapatos de calidad a un precio razonable, etc. Lo esencial del concepto de «bienes internos de una institución» es que se refiere a algún tipo de servicio que se presta a los usuarios de la misma; la idea clave es que los bienes internos son los que dan sentido a la existencia misma de la institución, los que la legitiman ante la sociedad, los que hacen que la institución sea algo deseable y amable, puesto que, si no fuera por ella, algunas necesidades humanas quedarían sin satisfacer.

En el caso de la Universidad, generalmente nos referimos a sus bienes internos en términos de:

1) «Formación de alta calidad», 2) «investigación de excelencia» y 3) «transferenciaextensión de conocimientos a la sociedad». Para el logro de estas tres metas se precisa el apoyo de profesionales de la docencia y la investigación (profesorado) y también de profesionales de la gestión y la administración (personal de administración y servicios), pero todos con la mirada puesta en los beneficiarios de la actividad universitaria, que son, en primer lugar, el alumnado, y en segundo lugar los empleadores de los egresados, las empresas que acogen la transferencia de investigación y la sociedad en general.

Por otro lado, como ya anunciamos, además de los bienes internos, existe otra clase de bienes relacionados con las instituciones: los llamados «bienes externos», es 
decir, las recompensas que recibe la institución y sus miembros como compensación por el servicio que presta. Se trata de los ingresos económicos, el poder, la influencia, la fama, los premios y reconocimientos, los honores, el prestigio, la buena imagen social, etc. Por ejemplo, la profesión médica percibe honorarios, prestigio, poder, influencia, etc., cuando realiza su cometido (bienes internos) del modo adecuado. La profesión periodística, por su parte, puede tener ingresos, poder, fama, prestigio, buena reputación, etc. La profesión docente recibe generalmente un sueldo, un reconocimiento, cierta buena imagen, prestigio, etc. Una organización empresarial del tipo “fábrica de zapatos” puede conseguir dividendos para el accionista, fama, buena imagen de marca, etc. En el caso de la institución universitaria, los bienes externos suelen ser: ingresos, prestigio, poder, fama, buena reputación, valoración social de los títulos que expide, etc.

Ahora bien, ¿qué ocurre cuando una institución (o una persona que labora en ella) pone por delante la consecución de bienes externos a toda costa y por cualquier medio, en lugar de otorgar la debida prioridad al logro de los bienes internos, que son los que legitiman la existencia misma de la institución? Lo que ocurre en estos casos es que la institución (o la persona que labora en ella) incurren en un caso de corrupción (Cortina 1996). Porque la corrupción, desde el punto de vista del funcionamiento institucional, se puede comprender como una desviación respecto a las metas que dan sentido a la institución de que se trate. Por ejemplo, si una persona se matricula en una Universidad, por el solo hecho de hacerlo tiene la expectativa legítima de recibir una formación de calidad en aquello en lo que se ha matriculado. Por eso, si tal universidad no responde adecuadamente a esa expectativa legítima por causas imputables a su responsabilidad como institución, se puede decir que dicha universidad se ha corrompido, puesto que está fallando gravemente en cuanto a proporcionar el servicio que se ha comprometido a prestar a su alumnado. Desde el punto de vista ético, la corrupción no se limita a los casos 
de soborno, apropiación indebida y casos semejantes, sino que incluye también, de modo más general, cualquier desviación culpable con respecto a la prioridad que debe otorgarse a los bienes internos de la institución.

Una vez realizada la distinción conceptual entre los bienes internos y externos de la institución universitaria, pueden quedar claras las razones por las que proponemos, como principios éticos básicos de la institución universitaria los siguientes:

1) La Universidad es una institución social radicada en una sociedad comprometida con el respeto de la dignidad de las personas, de modo que su primer deber es respetar y promover los derechos humanos de todas las personas que tienen relación con ella (alumnado, profesorado, administrativos, proveedores, etc.).

2) Todos los miembros de la comunidad universitaria deben otorgar prioridad a la producción de los bienes internos de la institución, en lugar de buscar directamente los bienes externos descuidando el logro de los primeros.

3) Se debe buscar honestamente la satisfacción de todos los grupos de afectados por la institución, haciendo compatibles sus legítimos intereses en la medida de lo posible: el alumnado, la comunidad a la que se presta el servicio (empleadores de egresados, empresas a las que se transfieren resultados de investigación, etc.), los empleados (profesorado, personal de administración y servicios), los proveedores, etc.

Estos principios se pueden concretar por medio de códigos éticos, códigos de buenas prácticas y otros documentos semejantes ${ }^{3}$. Pero lo esencial es la formación ética

\footnotetext{
${ }^{3}$ Muchas Universidades españolas se han dotado de un código ético. Recientemente tuve ocasión de colaborar en la elaboración del que tiene la Universidad Politécnica de Cartagena (UPCT). Como se explica en el anexo del mismo, la elaboración participativa es una condición necesaria, aunque no suficiente por
} 
de las personas que conforman la comunidad universitaria, de modo que se vaya creando una cultura corporativa que encarne esos tres principios como lo habitual y mayoritario, con independencia de que siempre habrá algunos casos puntuales de falta de compromiso ético. A continuación vamos a comentar algunos de los fallos éticos más habituales en nuestras Universidades, tratando de mostrar las razones por las que se trata de malas prácticas que conviene erradicar.

\section{Algunos problemas éticos que socavan la ética universitaria}

\subsection{Algunos problemas éticos que afectan al personal docente e investigador}

- Competencia desleal con empresas. En ocasiones, se disfraza la realización de actividades privadas con ánimo de lucro de sus promotores como si fueran contratos de investigación y transferencia de conocimiento de los que están previstos en la legislación vigente. Por ejemplo: una empresa contrata con un grupo de investigadores universitarios la realización de un estudio de opinión sobre cierto producto que comercializa dicha empresa; pero ni la empresa ni el grupo investigador tienen en cuenta que ya existen gabinetes de investigación que realizan este tipo de estudios con todas las garantías de rigor científico, pero que no pueden competir en precios con los que ofrecen los universitarios, dado que éstos tienen unas infraestructuras y unos ingresos principales que les proporciona la universidad. En este caso se observa una violación del tercer principio mencionado anteriormente,

sí sola, para que el código sea asumido en la práctica por todos sus destinatarios. Puede consultarse en: https://transparencia.upct.es/?page_id=583 
puesto que no se están armonizando adecuadamente los legítimos intereses de todos los colectivos afectados.

- Abusos en autoría de trabajos. Por ejemplo: hay directores de tesis doctorales que siguen apareciendo como coautores de los trabajos publicados mucho después de la defensa de la tesis. Esto puede interpretarse como una falta de atención al segundo principio, puesto que los directores de tesis que obligan a sus pupilos a compartir la autoría de trabajos, tras un tiempo prudencial en el que esa práctica ha tenido sentido, estarían poniendo su afán de acumular bienes externos (en este caso méritos de investigación que en realidad ya no les corresponden) por delante de los bienes internos de la actividad investigadora (en este caso, proporcionar mediante el propio esfuerzo una investigación de calidad).

- Apropiación de obras ajenas. Por ejemplo, algunos investigadores se han apropiado de trabajos de compañeros, e incluso de alumnos. Esta falta de cuidado con los derechos de autor es mucho más grave que la anterior. Porque ahora ya no se trata de mantenerse como coautor en trabajos en los que inicialmente sí que hubo cierta coautoría, sino que se trata, lisa y llanamente, de un robo. Esto atenta contra el primer principio ético reseñado, puesto que viola derechos básicos de las personas.

- Corruptelas en proyectos financiados. Por ejemplo, se consiguen facturas para justificar gastos que no se han hecho y se hacen viajes privados con el dinero del proyecto, a costa de la calidad de los resultados. En este tipo de casos también se estaría otorgando prioridad a los intereses particulares del investigador frente al 
imperativo ético de otorgar la prioridad a la calidad de la investigación. Por lo tanto, se viola el primer principio.

- Falta de atención a la docencia. En ocasiones hay profesorado universitario que, descuida gravemente la docencia en beneficio de la investigación. La profesión de profesor o profesora de universidad lleva consigo, necesariamente, la condición de docente. Esto no es opcional, puesto que las universidades tienen alumnado y este debe ser adecuadamente formado. Sin embargo, en los años recientes se ha estado incentivando mucho más la investigación (sexenios, proyectos financiados, méritos que realmente cuentan en los concursos de acceso, etc.) que la docencia. Pero esto no debería ser así. Aunque la universidad tiene autonomía para repartir las tareas entre sus miembros, nunca debería permitir que haya profesorado exclusivamente investigador ni exclusivamente docente, porque la figura de profesor o profesora de universidad lleva consigo las dos tareas, que se complementan y se necesitan mutuamente. Descuidar la docencia es descuidar uno de los bienes internos a los que se refiere el segundo principio.

- Represalias a estudiantes que se han atrevido a quejarse de alguna cosa que hace el profesor o profesora. Aquí nos referimos exclusivamente a las situaciones injustas en las que el ejercicio del poder académico por parte del docente (particularmente en lo que se refiere a las evaluaciones y calificaciones del rendimiento del alumnado para acreditar si ha alcanzado las competencias que debería alcanzar) se extralimita claramente. Esto se puede comprobar, hasta cierto punto, analizando si se han cumplido adecuadamente las reglas de juego de la docencia (guía docente de la materia o asignatura, normativa del departamento, etc.), de modo que no se interprete 
como represalia la simple aplicación de los criterios establecidos. Pero si realmente hay un abuso por parte del docente, entonces se estaría violando el primer principio, puesto que no se estarían respetando algunos derechos básicos del alumnado.

- Absentismo de profesorado. Hay ocasiones en los que el docente titular de una materia o asignatura se ausenta excesivamente por diversos motivos (algunos pueden ser legítimos y otros no) y no siempre se avisa al alumnado con la debida antelación y por los canales más seguros; en ocasiones, la mayor parte de la docencia se deja a cargo de algún becario o becaria sin supervisión alguna de si lo hace bien o mal. El resultado de todo ello es que el alumnado, que tenía unas expectativas legítimas de recibir la mejor formación posible, finalmente no logra ver colmadas tales expectativas. En muchos de estos casos se estaría violando el principio primero (falta de respeto al alumnado), y en parte también el segundo (no se otorga prioridad a uno de los bienes internos de la universidad) y también el tercero (no se compaginan adecuadamente los intereses legítimos de unos y otros colectivos).

- Abusos en la evaluación. Por ejemplo, algunas veces el alumnado se queja de que no hay criterios claros en los procesos de evaluación, de no se cumplen los horarios de atención en las tutorías, no se lleva a cabo la preceptiva oportunidad de revisión de exámenes y tareas académicas, etc. Esto se puede interpretar éticamente como una grave falta de respeto al alumnado (principio primero) y también como una postergación del bien interno de la docencia frente al acopio de bienes externos (por ejemplo, por lo general el profesorado que comete este tipo de abusos sigue cobrando lo mismo y teniendo el mismo poder, aunque tal vez se resienta su imagen pública o prestigio profesional). 
- No corregir las tareas del alumnado. Esta corruptela es muy perjudicial para el alumnado, porque los estudiantes acaban considerando como correcto lo que en realidad es incorrecto. En la tarea docente, la retroalimentación sobre las tareas que hace el alumnado es un elemento básico del proceso de enseñanza-aprendizaje. Por lo general, este fallo lo cometen docentes que, o bien se dejan llevar por la pereza (lo cual es una falta de respeto al alumnado, contra el principio primero), o bien se ven sobrecargados de tareas por falta de previsión o mala gestión de su tiempo (en cuyo caso, se estaría faltando al tercer principio, por negligencia profesional en cuanto a procurar la adecuada compatibilidad de legítimos intereses de todos los afectados).

\subsection{Algunos problemas éticos que afectan al personal de administración y} servicios y al profesorado que asume tareas de gestión

- Falta de confidencialidad. En ocasiones se difunden datos personales, relatos y rumores acerca de miembros de la comunidad universitaria de un modo irresponsable y éticamente incorrecto. Este tipo de comportamientos es perjudicial para el logro de los bienes internos de la institución. En consecuencia, además de violar el principio primero (falta de respeto a las personas), también viola el segundo principio (falta de prioridad a los bienes internos de la Universidad).

- Falta de flexibilidad. En ocasiones se gestionan los procedimientos burocráticos con una rigidez que es tan excesiva que provoca graves daños a las personas. Por lo general, las normas han de ser interpretadas para poder ser aplicadas, y no siempre la interpretación más favorable a la parte más débil es la que se adopta como válida. 
Pero la necesaria flexibilidad no está reñida con la justicia, sino todo lo contrario: para que haya verdadera justicia se precisa que se apliquen las normas con cierta flexibilidad. Si se carece de ella, se puede incurrir en violaciones de derechos básicos (principio primero) o también en faltas contra la necesaria compatibilización entre los derechos de las personas afectadas (principio tercero).

- Falta de eficiencia en el uso de los recursos. Como los recursos son limitados y generalmente los proporcionan los contribuyentes a través de los impuestos (en el caso de las universidades públicas), existe un deber moral de utilizarlos con eficiencia, es decir, tratando de obtener el mayor rendimiento con la menor cantidad posible de recursos. El despilfarro es un fallo moral grave, porque a menudo afectará a los derechos básicos de las personas (por ejemplo, eliminando becas o puestos de trabajo), atenta contra la prioridad de los bienes internos y generalmente afecta también a la necesaria compatibilidad entre los intereses legítimos de los grupos y personas.

- Falta de equidad en contrataciones de profesorado y de personal de administración y servicios. Todavía son relativamente frecuentes las situaciones de enchufismo en los temas de contratación, el clientelismo y el amiguismo. Esto atenta contra los derechos básicos de las personas (principio primero), contra la debida prioridad de los bienes internos sobre otros intereses (principio segundo) y también contra la posible compatibilización de expectativas legítimas de los colectivos universitarios (por ejemplo, la legítima expectativa del alumnado de que se contrate al mejor docente posible para ocupar una vacante, quedaría frustrada si se acaba contratando a alguien con menor cualificación pero que ocupaba interinamente el puesto). 
- Sensación de impunidad. En ocasiones, cunde la desmoralización de los miembros de la comunidad universitaria ante la impunidad que se observa en determinados casos de falta de profesionalidad. Especialmente cuando se observa que a quien no cumple con sus obligaciones universitarias no se le penaliza con un menor acceso a los bienes externos (recordemos: ingresos, poder, buena imagen, etc.). Esta desmoralización es muy dañina para la universidad, porque corroe el carácter de sus miembros hasta el punto de que puede hacer cundir la desidia, el cinismo, la chapuza, el disimulo y la corrupción general. La impunidad es sobre todo una falta de respeto contra el segundo principio, puesto que atenta contra la prioridad de los bienes internos frente al reclamo de recompensas merecidas. Si se da el mismo trato a quien no aporta los bienes internos en la proporción que le corresponde que a quien no lo hace, se favorece al corrupto frente al excelente y al correct, provocando una situación claramente injusta.

- Falta de transparencia y rendición de cuentas. En ocasiones no está accesible para el público la información que debiera estar disponible. Esto provoca desconfianza, sospechas de posible corrupción y la consiguiente desmoralización corrosiva. Ocultar información que debiera ser accesible atenta contra los tres principios que estamos comentando, puesto que afecta a derechos básicos de las personas, perjudica el logro de los bienes internos de la universidad y dificulta la compatibilización de las expectativas legítimas de los colectivos relacionados con la institución. Por otra parte, no se debe olvidar que la gestión de los conflictos ha de tener también una parte pública: aunque sea necesaria la confidencialidad para poder alcanzar acuerdos, también es necesaria la transparencia para que tales acuerdos sean legítimos. 
- Casos de discriminación por razón de género. Aunque ha habido importantes avances en este terreno, existen todavía algunas actitudes de cierto descrédito con respecto a las aportaciones femeninas e injusticia en la distribución de cargos en la Universidad. Este tipo de casos atentan claramente contra el primer principio, puesto que afectan a los derechos básicos de las personas.

\subsection{Algunos problemas éticos que afectan al alumnado}

- Falta de respeto a otros estudiantes, al profesorado y al personal de administración y servicios. A veces nos encontramos con algunos estudiantes que claramente no respetan las formas elementales de trato justo a las personas. Esto debe ser corregido con medidas educativas y, en casos extraordinarios, con medidas punitivas. En estos casos se trata de una violación del principio primero que, al mismo tiempo, dificulta el cumplimiento de los otros dos principios.

- Falta formación ética en los egresados. En ocasiones los empleadores de egresados universitarios se quejan de que éstos presentan ciertas carencias, como la falta de una cultura general (complementaria de la formación específica que hayan recibido en la carrera) y la ausencia de capacidades para trabajar en equipo. Este fallo indica que no se está tomando muy en serio el segundo principio (prioridad de los bienes internos de la universidad). Para afrontar este problema, es obvio que tanto la universidad como los propios estudiantes han de poner de su parte para avanzar hacia el logro de una completa y excelente formación del alumnado. 
- Plagio en los trabajos académicos. Este fallo moral afecta a los derechos de autor de los textos plagiados, además de que provoca el engaño del lector desprevenido, que tomará por obra del plagiador lo que en realidad es obra de otras personas. Por tanto, se está faltando al respeto de los autores a quienes se toma la información sin citar la fuente, como también se falta al respeto de los lectores del trabajo académico, a quienes se pretende engañar en cuanto a la autoría del texto. Todo ello viola el primer principio (derechos básicos de las personas) y afecta muy negativamente al cumplimiento de los otros dos.

- Falta de esfuerzo y vocación. Se ha detectado en un sector del alumnado la falta de una cultura del esfuerzo, del trabajo bien hecho, del ánimo de aprender. En su lugar, se ha instalado en algunos la abulia, el desprecio del saber y la ausencia de compromiso con los valores universitarios. Este fallo moral supone un desafío muy importante para la universidad, porque seguramente no es ella quien ha provocado la existencia de un colectivo juvenil que presenta estas carencias. Sin embargo, en la medida en que la universidad tiene que responder a los retos de la época en la que vive, no puede mirar para otro lado e ignorar este tipo de problemas. Desde el punto de vista ético, sería importante promover actividades universitarias especialmente diseñadas para llegar a este grupo de personas y promover en ellas un cambio de mentalidad. De ese modo, la Universidad no solo estará velando por la mejor formación posible de este sector estudiantil, sino que también estará trabajando por mejorar el clima general de toda la institución, promoviendo los bienes internos que le corresponden (principio segundo) y ayudando a que los otros dos principios se tomen más en serio. 


\section{4. ¿Qué se puede hacer para promocionar la ética en las universidades del siglo}

XXI?

Una de las cosas más elementales que se puede hacer para promocionar la ética en las universidades es elaborar de manera participativa un código ético. En dicho código se destacarán unos pocos valores que cada universidad considere como urgentes y prioritarios en el momento histórico en el que está viviendo; cada valor suele ir acompañado de un conjunto de buenas prácticas que lo explicitan y que van encaminadas a orientar los comportamientos concretos de los colectivos universitarios, con el fin de animarlos a promover los bienes internos de la institución.

Una vez que se dispone del código ético, es trascendental llevar a cabo un seguimiento del mismo. Pero este seguimiento no consiste en vigilar y castigar, puesto que el código ético no es un reglamento que contenga sanciones de ningún tipo. El seguimiento, llevado a cabo por medio de una comisión ad hoc, de la que suele formar parte el Defensor o Defensora del universitario, se centra más bien en la difusión del código, la organización de actividades formativas relacionadas con valores éticos, y en general, la promoción del conocimiento y la adhesión voluntaria a los contenidos del código ético. Esta comisión de seguimiento del código ético podría atender también, si se estima oportuno, las consultas de tipo ético que pueda plantear cualquier miembro de la comunidad universitaria, de modo similar a como ya se viene haciendo en muchos hospitales en los que funciona un comité de ética asistencial. La composición de una comisión de seguimiento del código ético, para que fuese realmente operativa, debería contar con un número reducido de personas (no más de diez) y debe tener características como las siguientes: 
- Ha de tener representación de los colectivos universitarios (docentes, alumnado, personal de administración y servicios, consejo social);

- ha de ser independiente del equipo de gobierno de la universidad;

- ha de contar con algún especialista en Ética;

- ha de contar con el apoyo técnico y presupuestario que sea preciso para garantizar su funcionamiento óptimo;

- ha de reunirse con la debida regularidad (al menos una vez al semestre) y para garantizar que haya quorum se ha de prever que haya miembros titulares y suplentes;

- ha de tener un reconocimiento oficial en los Estatutos de la Universidad, de modo que a sus miembros se les compute el trabajo realizado en la comisión como parte de su jornada laboral ordinaria;

- la comisión ha de tener su propio reglamento de funcionamiento interno, compromiso de confidencialidad en las deliberaciones, levantamiento de actas, etc.

En segundo lugar, otra cosa que se puede hacer para promocionar la ética en las universidades es tomar medidas para evitar las situaciones de impunidad en aquellos casos en los que se observa la vulneración de los derechos de las personas. Esto significa que es preciso fomentar una cultura interna en la que no exista miedo o desidia para denunciar las malas prácticas, así como medios suficientes para una adecuada investigación e inspección de los hechos, revisión de las normas vigentes, etc. Es esencial que se estimule la cultura de la denuncia respetuosa y responsable, frente a la cultura del compadreo, de la simulación y de la resignación. Y también es imprescindible mejorar los reglamentos para evitar aquello de "hecha la ley, hecha la trampa". Los reglamentos 
han de ser razonables y ha de ser viable su cumplimiento en condiciones normales, de modo que se pueda controlar su incumplimiento con facilidad.

En tercer lugar, pero no menos importante, se puede revisar el sistema de incentivos (los bienes externos que reciben las personas conforme al puesto que ocupan en la universidad), de modo que sea eficaz para promover los bienes internos, que siempre han de ser lo prioritario. Por ejemplo: ¿se ha de conceder un quinquenio de docencia a todo el que lo pida, sin tener que mostrar que se ha llevado a cabo una buena labor docente? ¿Se puede incentivar más la docencia, como en su día se hizo con la investigación al crear los sexenios? Etc. El establecimiento de un buen sistema de incentivos es clave para el cumplimiento del tercer principio ético que hemos mencionado anteriormente: buscar honestamente la satisfacción de todos los grupos de afectados por la institución, haciendo compatibles sus legítimos intereses en la medida de lo posible. El éxito o el fracaso de la ética universitaria, a mi juicio, radica especialmente en el ajustamiento del sistema de incentivos, tanto positivos (estímulos, reconocimientos, complementos retributivos, calendario y horario laboral, etc.), como negativos (penalizaciones de diverso tipo). Porque el sistema de incentivos es el que crea, a medio y largo plazo, una cierta rutina, una cultura de la vida cotidiana, de modo que, si queremos una universidad ética, hemos de crear una cultura interna de adhesión a los valores éticos, estimulando las buenas prácticas y desincentivando las que nos perjudican. 


\section{Referencias bibliográficas}

Cortina, A. (1993): Ética aplicada y democracia radical. Madrid: Tecnos.

Cortina, A. (1996): «La regeneración moral de la sociedad y de la vida política» en Cortina, A. y otros: Corrupción y ética. Bilbao: Universidad de Deusto, pp. 29-37.

Cortina, A. y Martínez, E. (1996): Ética. Madrid: Akal.

MacIntyre, A. (1981): After virtue: a study in moral theory. London: Duckworth. Hay trad. esp. de Amelia Valcárcel (1987): Tras la virtud. Barcelona: Crítica.

Martínez Navarro, E. (2010): Ética profesional de los profesores. Bilbao: Desclée de Brouwer. 